\title{
Detection of SARS-CoV-2 Specific Memory B cells to Delineate Long-Term COVID-19 Immunity
}

\author{
Constantin Thieme ${ }^{1}$, Mohamed Abou-el-Enein ${ }^{1}$, Enrico Fritsche ${ }^{1}$, Moritz Anft ${ }^{2}$, Sarah \\ Skrzypczyk $^{2}$, Adrian-Atila-Nicolas Doevelaar ${ }^{2}$, Magdi Elsallab $^{1}$, Nicola Brindle ${ }^{1}$, \\ Krystallenia Paniskaki ${ }^{3}$, Felix Seibert ${ }^{2}$, Oliver Witzke ${ }^{3}$, Timm Westhoff ${ }^{2}$, Ulrik Stervbo ${ }^{2}$, \\ Guido Heine ${ }^{4}$, Toralf Roch ${ }^{1}$, and Nina Babel ${ }^{1}$ \\ ${ }^{1}$ Charité Universitätsmedizin Berlin \\ ${ }^{2}$ Marien Hospital Herne Academic Teaching Hospital of the University Bochum \\ ${ }^{3}$ University Hospital Essen \\ ${ }^{4}$ University Hospital Schleswig Holstein
}

January 15, 2021

\begin{abstract}
Background: The ongoing COVID-19 pandemic, caused by the novel coronavirus SARS-CoV-2, represents a serious worldwide health concern. A deeper understanding of the immune response to SARS-CoV-2 will be required to refine vaccine development and efficacy as well as to evaluate long-term immunity in convalescent patients. With this in mind, we investigated the formation of SARS-CoV-2 specific BMEMORY cells from patient blood samples. Methods: A standard flow cytometry-based protocol for the detection of SARS-CoV-2 specific B cells was applied using fluorochrome-coupled SARS-CoV-2 spike (S) full-length protein. Cohorts of 26 central European convalescent mild/moderate COVID-19 patients and 14 healthy donors were assessed for the levels of SARS-CoV-2 S- specific BMEMORY cells. Results: Overall B cell composition was not affected by SARS-CoV-2 infection in convalescent patients. Our analysis of SARS-CoV-2 specific BMEMORY cells in samples collected at different time points revealed that S-protein specific B cells remain in peripheral blood at least up to 6 months after COVID-19 diagnosis. Conclusions: Detection of SARS-CoV-2 specific BMEMORY cells may improve our understanding of the long-term adaptive immunity in response to SARS-CoV-2, allowing for an improved public health response and vaccine development during the COVID-19 pandemic. Further validation of the study in larger and more diverse populations and a more extended observation period will be required.
\end{abstract}

\section{Detection of SARS-CoV-2 Specific Memory B cells to Delineate Long-Term COVID-19 Im- munity}

\section{Short title: Long-term SARS-CoV-2 Specific Memory B cells}

Constantin J. Thieme ${ }^{1 *}$, Mohamed Abou-el-Enein ${ }^{1,2,3 *}$, Enrico Fritsche ${ }^{1}$, Moritz Anft ${ }^{4}$, Sarah Skrzypczyk ${ }^{4}$, Adrian Doevelaar ${ }^{5}$, Magdi Elsallab ${ }^{1,3}$, Nicola Brindle ${ }^{1}$, Krystallenia Paniskaki ${ }^{6}$, Felix S. Seibert ${ }^{5}$, Oliver Witzke $^{6}$, Timm H. Westhoff ${ }^{5}$, Ulrik Stervbo ${ }^{4}$, Guido Heine ${ }^{7 *}$, Toralf Roch $^{1,4 *}$, Nina Babel ${ }^{1,4 *}$

${ }^{1}$ Charité - Universitätsmedizin Berlin, Corporate Member of Freie Universität Berlin, Humboldt-Universität zu Berlin, and Berlin Institute of Health (BIH), BIH Center for Regenerative Therapies, and Institute of Medical Immunology, Augustenburger Platz 1, 13353 Berlin, Germany

${ }^{2}$ Division of Medical Oncology, Department of Medicine, and Department of Stem Cell Biology and Regenerative Medicine, Keck School of Medicine, University of Southern California, Los Angeles, CA USA 
${ }^{3}$ Cell therapy program, Keck School of Medicine, University of Southern California, and Children Hospital Los Angeles (CHLA), Los Angeles, CA USA

${ }^{4}$ Center for Translational Medicine and Immune Diagnostics Laboratory, Medical Department I, Marien Hospital Herne, University Hospital of the Ruhr-University Bochum, Hölkeskampring 40, 44625 Herne, Germany

${ }^{5}$ Medical Department I, Marien Hospital Herne, University Hospital of the Ruhr-University Bochum, Hölkeskampring 40, 44625 Herne, Germany

${ }^{6}$ Department of Infectious Diseases, West German Centre of Infectious Diseases, University Hospital Essen, University Duisburg-Essen, Germany

${ }^{7}$ Department of Dermatology and Allergy, University Hospital Schleswig Holstein, Arnold-Heller-Straße 3, 24105 Kiel, Germany

*equal contribution

Word count (main text) : 2501

Acknowledgments: We are grateful to the patients who donated their blood samples and clinical data for this project. We would like to acknowledge the excellent technical assistance as well as the expertise of the immune diagnostic laboratory of the Center for Translational Medicine at Marien Hospital Herne. N.B. received funding from the Mercator Foundation, the German Federal Ministry of Education and Research (BMBF) e:KID (01ZX1612A), and BMBF NoChro (FKZ 13GW0338B). MA received funding from the Arab German Young Academy (AGYA), a project by the BMBF.

\section{Abstract}

Background: The ongoing COVID-19 pandemic, caused by the novel coronavirus SARS-CoV-2, represents a serious worldwide health concern. A deeper understanding of the immune response to SARS-CoV-2 will be required to refine vaccine development and efficacy as well as to evaluate long-term immunity in convalescent patients. With this in mind, we investigated the formation of SARS-CoV-2 specific BMEMORY cells from patient blood samples.

Methods: A standard flow cytometry-based protocol for the detection of SARS-CoV-2 specific B cells was applied using fluorochrome-coupled SARS-CoV-2 spike (S) full-length protein. Cohorts of 26 central European convalescent mild/moderate COVID-19 patients and 14 healthy donors were assessed for the levels of SARS-CoV-2 S- specific B BeMORY cells.

Results: Overall B cell composition was not affected by SARS-CoV-2 infection in convalescent patients. Our analysis of SARS-CoV-2 specific B BEMORY cells in samples collected at different time points revealed that S-protein specific B cells remain in peripheral blood at least up to 6 months after COVID-19 diagnosis.

Conclusions: Detection of SARS-CoV-2 specific B BEMORY cells may improve our understanding of the long-term adaptive immunity in response to SARS-CoV-2, allowing for an improved public health response and vaccine development during the COVID-19 pandemic. Further validation of the study in larger and more diverse populations and a more extended observation period will be required.

Keywords : COVID-19, Long-term immunity, Memory B cells, SARS-CoV-2

\section{Introduction}

The COVID-19 pandemic has led to devastating health outcomes with the death toll exceeding one million cases by the end of 2020. Furthermore, the global lockdowns impeded social and commercial interactions, with psychological and economic impacts on the public ${ }^{1}$. Vaccines are currently in the final stages of approval or have already been authorized under emergency use authorization ${ }^{2}$. However, there is still limited data on long-term immunity against SARS-CoV-2 ${ }^{3}$. Studying long term immunity is essential to aid decision making for reopening and easing social distancing, assess the need for booster doses of vaccines, and estimate the potential of reinfections ${ }^{4,5}$. Focusing mainly on antibody-based assays as readouts for long-term immunity 
may be strongly impeded due to lack of consistency in circulating antibody levels regardless of disease severity. Indeed, there are conflicting results about the longevity of antibody response in SARS-CoV-2 infected individuals ${ }^{6-10}$, suggesting that long-lived plasma cells may not have been efficiently established in a proportion of COVID-19 patients. Another study found that about $20 \%$ of German SARS-CoV-2 PCRpositive patients with mild to moderate symptoms did not have detectable spike-specific antibodies when tested 3 weeks or longer after infection ${ }^{11}$. In line with these findings, Long et al. reported that $40 \%$ of nonsymptomatic patients, as well as $12.9 \%$ with symptoms, became seronegative with no detectable antibodies in the blood eight weeks after discharge from hospital ${ }^{12}$. Therefore, it is of uttermost importance to develop and test other diagnostic assays to study the long-term immunity against SARS-CoV-2.

For diagnostic assays, the right target, the test accuracy, and minimizing false-negative and false-positive outcomes are the major challenges ${ }^{13}$. One potential approach that can help to improve the detection of longterm immunity is the analysis of SARS-CoV-2-specific memory $\mathrm{T}$ and $\mathrm{B}$ cell formation. We and others have reported on the detection of a strong SARS-CoV-2 specific memory $\mathrm{T}$ cell response in immune-competent and immunosuppressed patients ${ }^{14-18}$. Similar studies of B cell-related immunity have so far focused mainly on characterizing antibody specificities ${ }^{19-24}$. Other studies identified and isolated SARS-COV-2 B cells to generate neutralizing antibodies or to characterize the $\mathrm{B}$ cell receptor usage ${ }^{20-22}$. $\mathrm{B}_{\mathrm{MEMORY}}$ cells can remain for decades or potentially lifelong, located within lymph nodes, spleen, bone marrow, and the lung, or circulate in the blood ${ }^{25-28}$. Upon reinfection, they become re-activated by encountering the same antigen and immediately start to proliferate and differentiate into plasma cells secreting neutralizing antibodies. Thus, quantifying $\mathrm{B}_{\mathrm{MEMORY}}$ cell levels by a reliable assay may be used as an indicator of long-term immunity in convalescent patients. Although the formation of $\mathrm{B}_{\mathrm{MEMORY}}$ cells was described, controlled studies comparing SARS-COV-2 specific memory B cell formation in unexposed individuals and COVID 19 patients are limited. To our knowledge, only studies with Australian and US-American cohorts assessed the long-term immunity through characterization of the $\mathrm{B}_{\text {MEMORY cells for more than } 6 \text { months }}{ }^{29,30}$. Since the reports on duration of S1-specific antibody detection upon SARS-CoV-2 resolution are conflicting, several studies on antibodyproducing $\mathrm{B}_{\mathrm{MEMORY}}$ cells performed within different ethnic origin populations are required. In this work, we focus on detecting SARS-CoV2 spike (S)-protein specific memory B cells (BMEMORY) in a well characterized cohort of central European COVID-19 patients as compared to unexposed controls. Furthermore, we analysed the sustainability for several months after infection as a specific long-term marker of adaptive immunity.

\section{Methods}

\section{Protocol development}

Well established and tested approaches were adapted for ease and simplicity ${ }^{31-33}$. A schematic presentation of the protocol is depicted in Figure 1.

In brief, blood was collected using EDTA coated Vacuette ${ }^{\circledR}$ tubes. Peripheral blood mononuclear cells (PBMCs) were isolated by density gradient centrifugation technique with BioColl separating solution permitting cell separation based on polysucrose polymers. Short-term cell culture maintenance was performed by Roswell Parm Memorial Institute (RPMI) 1640 medium supplemented with GlutaMAX ${ }^{\mathrm{TM}}$, 1\% Penicillin/ Streptomycin, and $10 \%$ fetal bovine serum (FBS). For flow cytometric screening of human samples, SARSCoV-2 spike protein was labeled with fluorescein (FITC) or Cy5 fluorochromes using Lightning-Link (B) rapid labeling kits. This allows direct linkage of SARS-CoV-2 specific S- protein by robust covalent binding and fast processing of the assay as corresponding conjugates are ready-to-use in less than twenty minutes. Labeling the protein of interest independently with two different fluorochromes before incubation of the sample and analyzing the double-positive cells greatly enhances the specificity ${ }^{31,32}$. Subsequently, only double-positive B cells binding S-FITC and S-Cy5 were subjected to further evaluations. Another control measure to ensure specificity is to demonstrate that the response can be blocked by excess unlabelled SARS-CoV-2 S-protein, which blocks the S-protein specific B-cell receptors, thereby inhibiting the binding of the S-FITC and S-Cy5 to the B cells ${ }^{33}$.

A detailed list of all materials and reagents, as well as devices used, is provided in Table 1. 
We designed a staining panel to enable the detection of SARS-CoV-2 specific B cells by applying a set of fluorophore-coupled antigens characteristic to different B cell subsets such as naïve, switched, and unswitched $\mathrm{B}_{\text {Memory }}$ cells. Detailed information on the panel is provided in Table 2.

Study participants / Validation cohorts

Between April and October 2020, a cohort of 26 convalescent COVID-19 patients and 14 healthy donors were included in the study in 3 German centres (Berlin, Bochum, Essen). Symptomatic patients with mild to moderate symptoms were eligible to participate at a median time of 48 days after onset of COVID19 symptoms (during which they become convalescent) according to the WHO management guidance (27 May 2020) and having a confirmed SARS-CoV-2 IgG ELISA at the time of study inclusion. All patients gave written informed consent. The ethical committee of the Ruhr-University Bochum approved the study (20-6886).

\section{Measurement of SARS-CoV-2 specific B cells}

Briefly, SARS-CoV-2 S1/S2-protein (further referred to as S-protein) (Sino Biological Inc.) was divided into three samples. Sample 1 was left unlabelled for blocking, sample 2 was coupled to fluorescein isothiocyanate (FITC), and sample 3 was linked to Cy5 fluorochrome. Lightning-Link ${ }^{\circledR}$ Rapid Conjugation System (Abcam PLC) was used for labelling according to the manufacturers' protocol. Labelling was performed with 100 $\mu \mathrm{g}$ of protein diluted to a concentration of $1 \mu \mathrm{g} / \mu \mathrm{L}$. After the labelling procedure, the protein integrity was checked by a gel electrophoresis. PBMC were divided into the respective samples (blocked and unblocked samples in concentrations of 1:500 and 1:1000 of fluorochrome-labelled S-protein) with 5x10 ${ }^{6}$ PBMC each (Fig. 1). Blocking was performed by using 10 the excess unlabelled protein. After blocking, PBMC were surface stained with fluorochrome-labelled antibodies, as indicated in Table 2. Finally, mixed FITC and Cy5 labelled protein was added in concentrations of 1:500 and 1:1000. Cells were stained for 10 min at $4{ }^{\circ} \mathrm{C}$. After washing with phosphate-buffered saline (PBS), samples were stored at $4{ }^{\circ} \mathrm{C}$ until measurement on a Cytoflex flow cytometer. Directly before analysis, the samples were stained with DAPI to differentiate live from dead cells. Further information on applied materials and equipment is provided in Table 1.

Measurement of anti-S IgG antibodies

Analysis of SARS-CoV-2 specific antibodies was performed using the SARS-Cov-2 IgG kit (EUROIMMUN $A G)$.

\section{Data analysis and statistical evaluation}

Flow cytometry data were analysed using FlowJo version 10.6.2 (BD Biosciences). Gating was performed according to single stains and fluorescent-minus-one controls. The corresponding gating strategy is presented in Figure 2. Lymphocyte identification and doublet exclusion were performed by forward-scatter (FSC) versus sideward parameters (SSC) and FSC/SSC-height (H) versus FSC/SSC-area (A) plotting, respectively. Cells positive for DAPI (dead cells), CD14 (mainly monocytes), or CD3 ( $\mathrm{T}$ cells) were excluded from further analyses. CD19 expressing cells were further characterized for IgD, CD27, and CD38 expression. Frequencies of SARS-CoV-2-protein specific B cells in recovered COVID-19 patients and healthy donors were compared using two-way repeated measurements ANOVA and Sidak's multiple comparisons test. Patient and healthy donor age was compared using unpaired two-tailed t-test, and gender was compared using two-tailed Fisher's exact test. Correlations were calculated using Spearman's rank coefficient. Statistical analysis and graphical representation were realized with GraphPad Prism v7 and R v3.6.2.

\section{Results}

\section{Baseline characteristics of the validation cohort}

The cohort comprised 26 convalescent COVID-19 patients and 14 healthy donors as a control group. The median age of the COVID-19 patients at the time of study inclusion was 59 years, with participants ranging from 27 to 88 years of age. Subjects were of mixed gender with a higher percentage of males (58\%) than females (42\%). COVID-19 patients were included at a median time of 48 days after diagnosis or onset of 
symptoms (range 15-214). Eighteen patients had mild COVID-19 that did not require hospitalization, and they did not receive specific COVID-19 treatment. Five patients had moderate disease, of whom two were hospitalized and one received treatment with antibiotics and remdesivir. Three patients had severe disease, were hospitalized and received antibiotic treatment. All included patients were confirmed to be SARS-CoV2 positive by RT-PCR and/or serological testing. Healthy volunteers (median age 48, range 26-60) were significantly younger than COVID-19 patients (median 59, range 27-88) $(P=0.0323$, two-tailed unpaired t-test). There were no significant differences regarding gender between COVID-19 patients and the control group ( $P=0.5024$, Fisher's exact test). Baseline characteristics are provided in Table 3.

Composition of $B$ cell subsets in convalescent COVID-19 patients and healthy controls

SARS-CoV-2 specific B cell response was analysed in COVID-19 patients and healthy donors by characterizing levels of IgD $\mathrm{CD}^{-} 7^{+} \mathrm{B}_{\mathrm{MEMORY}}, \mathrm{IgD}^{+} \mathrm{CD} 27^{+}$unswitched $\mathrm{B}_{\mathrm{MEMORY}}, \mathrm{IgD}^{+} \mathrm{CD} 27^{-} \mathrm{B}_{\mathrm{NAIVE}}$, and $\mathrm{CD} 27^{++}$ CD $38^{++}$plasmablasts (gating strategy shown in Figure 2).

Overall frequencies for all B cell subsets were similar between convalescent COVID-19 patients and healthy donors. $\mathrm{IgD}^{+} \mathrm{CD} 27^{-} \mathrm{B}_{\mathrm{NAIVE}}$ cells represented most $\mathrm{B}$ cells at around $50 \%$ of the population, while $\operatorname{IgD}{ }^{-}$ $\mathrm{CD} 27^{+} \mathrm{B}_{\text {MEMORYcells and } \mathrm{IgD}}{ }^{+} \mathrm{CD} 27^{+}$unswitched $\mathrm{B}_{\text {MEMORY }}$ cells demonstrated levels ranging between $15-20 \%$ of the entire B cell population. $\mathrm{CD} 27^{++} \mathrm{CD} 38^{++}$plasmablasts constituted on average less than 1 $\%$ of the B cells (Figure 3a).

Presence of SARS-CoV-2 S-protein specific BMEMORYcells in convalescent COVID-19 patients

Unlabelled SARS-CoV-2 S-protein in class-switched BMEMORY cells could significantly block binding in COVID-19 patients, but not healthy donor samples (Figure 2b, Figure 3c). SARS-CoV-2 specific B BEMORY cells correlated moderately with anti-S-protein IgG antibodies (Figure $3 \mathrm{~b}$ ). Overall, the frequency of Sprotein specific $\mathrm{B}_{\mathrm{MEMORY}}$ cells was significantly higher in the COVID-19 disease cohort compared to healthy individuals (Figure 3c). The difference between COVID-19 patients and healthy individuals was restricted to $B_{\text {MEMORY }}$ cells and was not observed for other B cell subsets such as naïve or unswitched $B_{\text {MEMORY cells }}$ (Figure 3d-f). This has two important implications: First, it demonstrates the specificity of the protocol for the detection of SARS-CoV-2 specific B BEMORY cells, since it is expected that only COVID-19 convalescent, but not unexposed individuals generate SARS-CoV-2 specific B cell memory. Second, it demonstrates that the humoral immune response in the analysed patient cohort could be mediated by long-lived memory B cells. We did not observe substantial amounts of SARS-CoV-2 specific plasmablasts (Figure 3e).

The longevity of B cell memory can also be demonstrated by analysing the frequency of SARS-CoV-2 specific

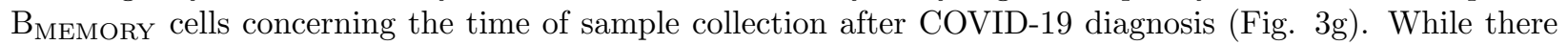
was a tendency of lower frequencies in samples collected at later time points, SARS-CoV-2 specific BMEMORY cells remained detectable also 6 months after COVID-19 diagnosis.

\section{Discussion}

In this study, we investigated the formation of S- protein-specific BMEMORY cells in convalescent COVID19 patients. We employed a control group to rule out significant cross-reactivity. Our findings indicated that there were minimal cross-reactive responses in healthy donors. Studying immunological memory and protection after SARS-CoV-2 infection is of particular importance for understanding the formation of B cell memory and, subsequently, the effectiveness of vaccination approaches as well as the need for further booster dosing. Thus, this study represents a step toward understanding COVID-19 long-term immunity.

Serological testing offers the potential for screening, contact tracing, evaluating the viral reservoir, and supporting epidemiologic assessments by detecting SARS-Cov-2 specific antibodies. Another challenge for a broad and robust application of antibody-based SARS-CoV-2 detection assays, especially in the long-term, is the currently questionable durability and high variability of the SARS-CoV-2 induced antibody responses, where neutralizing antibody titers decrease over time in SARS-CoV-2 infected individuals in some studies ${ }^{6-10}$. Furthermore, recent studies provided evidence that a proportion of SARS-CoV-2 infected individuals did not develop specific antibodies ${ }^{11,34,35}$. Thus, failed antibody formation or their rapid wane in circulation 
may limit our ability to verify COVID-19 immunity in the long-term, especially for mild or asymptomatic disease.

We, therefore, aimed at exploring immune responses to SARS-CoV-2 by studying and characterizing the $\mathrm{B}$ cell response. Such an approach enables us to understand long-term immunity more thoroughly. We

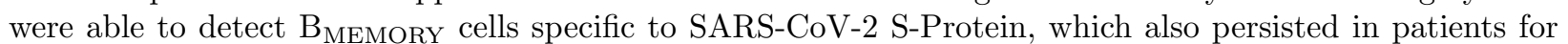
more than 200 days. B cells can provide serological memory for decades or potentially the entire lifespan of individuals ${ }^{25-27}$. Thus, although the antibodies that $\mathrm{B}$ cells produce during initial exposure may disappear within a few weeks, the generated $\mathrm{B}_{\mathrm{MEMORY}}$ cells can persist for much longer. For instance, previous studies were able to identify IgG memory B cells specific to the receptor-binding domain (RBD) in the blood of COVID-19 subjects ${ }^{22,24,29,36}$. Moreover, Zhang et al. demonstrated protective immunity formation with detectable levels of $\mathrm{B}_{\mathrm{MEMORY}}$ cells in a pediatric population ${ }^{37}$. Similarly, a recent study addressing SARSCoV-2 infection in rhesus macaques showed that two recovered subjects were resistant to reinfection one month later ${ }^{38}$. Our analysis of the S-protein SARS-CoV-2 specific B BEMORY cells in samples collected at different time points revealed that these cells remain in peripheral blood at least up to 6 months after COVID-19 diagnosis. Thus, our study performed on a central European cohort of patients is in line with the data on the recently published US American and Australian cohorts and thus, confirms and extends the knowledge on the B cell response against SARS-CoV- $2^{29,30}$. Therefore, our results demonstrating the longevity of SARS-CoV-2-specific B cell response can improve the general acceptance towards immunization in the broad community.

We acknowledge the limitations of our studies. First, only convalescent patients were studied, hence studying the B cell in patients with acute SARS-CoV-2 infection should be considered in further studies. Subsequent studies should also enroll larger patient cohorts with greater demographic variability and longer followup periods to decipher potential intra- and inter variability and to validate the finding that COVID-19 $B_{\text {MEMORY }}$ cells reflect a long-term immunity. Furthermore, our control cohort was significantly younger than the COVID-19 cohort, with a median difference of 10 years. However, this difference does not negatively affect the differences observed in SARS-CoV-2-specific BMEMORY in the two cohorts, since younger patients are supposed to have a stronger immune response. Nevertheless, we did not observe specific staining in the healthy donor cohort as evidenced by the lack of significant blocking of the staining.

In conclusion, our study evaluated the long-term immunity through the characterization of SARS-COV-2 specific $\mathrm{B}_{\text {MEMORY }}$ cells in a cohort of mild/moderate symptomatic COVID-19 patients versus healthy control subjects. We were able to detect S-specific B cells in the COVID-19 cohort even in mild cases. Overall, our study demonstrating the formation of SARS-CoV-2-specific long-term immunity may enable further opportunities for improving general vaccination acceptance thus supporting efforts to implement effective public health care measures during the COVID-19 pandemic.

\section{Conflict of interests}

All authors declare no competing interests.

\section{Authors' contributions}

C.J.T., M.Ab., T.R. and N.Ba. designed the study. CJT, M.An., S.S., K.P. performed the experiments. A.D., K.P., F.S.S., O.W., T.H.W. recruited patients. C.J.T., M.Ab, T.R. and N.Ba. analysed the data. C.J.T., M.Ab., E.F. and N.Ba. wrote the manuscript. M.An., N.Br., U.S., G.H., M.E reviewed and edited the manuscript. N.Br. supervised the project. All authors discussed the results and commented on the manuscript.

\section{References}

1. Nicola, M. et al. The socio-economic implications of the coronavirus pandemic (COVID-19): A review. International Journal of Surgery vol. 78 185-193 (2020).

2. Poland, G. A., Ovsyannikova, I. G. \& Kennedy, R. B. SARS-CoV-2 immunity: review and applications 
to phase 3 vaccine candidates. The Lancet vol. 396 1595-1606 (2020).

3. Vabret, N. et al. Immunology of COVID-19: Current State of the Science. Immunity vol. 52 910-941 (2020).

4. Silva-Cayetano, A. et al. A booster dose enhances immunogenicity of the COVID-19 vaccine candidate ChAdOx1 nCoV-19 in aged mice. Med (2020) doi:10.1016/j.medj.2020.12.006.

5. Tillett, R. L. et al. Genomic evidence for reinfection with SARS-CoV-2: a case study. Lancet Infect. Dis. 21, 52-58 (2020).

6. Gudbjartsson, D. F. et al. Humoral Immune Response to SARS-CoV-2 in Iceland. N. Engl. J. Med. 383 , 1724-1734 (2020).

7. Wajnberg, A. et al. Robust neutralizing antibodies to SARS-CoV-2 infection persist for months. Science (80-. ).370, eabd7728 (2020).

8. Seow, J. et al. Longitudinal observation and decline of neutralizing antibody responses in the three months following SARS-CoV-2 infection in humans. Nat. Microbiol. 5 , 1598-1607 (2020).

9. Prevost, J. et al. Cross-Sectional Evaluation of Humoral Responses against SARS-CoV-2 Spike. Cell Reports Med. 1, 100126 (2020).

10. Robbiani, D. F. et al. Convergent antibody responses to SARS-CoV-2 in convalescent individuals. Nature 584 , 437-442 (2020).

11. Solbach, W. et al. Antibody Profiling of COVID-19 Patients in an Urban Low-Incidence Region in Northern Germany. Front. Public Heal. 8 , 575 (2020).

12. Long, Q.-X. et al. Clinical and immunological assessment of asymptomatic SARS-CoV-2 infections. Nat. Med. (2020) doi:10.1038/s41591-020-0965-6.

13. Woloshin, S., Patel, N. \& Kesselheim, A. S. False Negative Tests for SARS-CoV-2 Infection — Challenges and Implications. N. Engl. J. Med. 383, e38 (2020).

14. Weiskopf, D. et al. Phenotype and kinetics of SARS-CoV-2-specific T cells in COVID-19 patients with acute respiratory distress syndrome. Sci. Immunol. 5 , (2020).

15. Grifoni, A. et al. Targets of T Cell Responses to SARS-CoV-2 Coronavirus in Humans with COVID-19 Disease and Unexposed Individuals. Cell 181 , 1489-1501.e15 (2020).

16. Babel, N. et al. Immune monitoring facilitates the clinical decision in multifocal COVID-19 of a pancreas-kidney transplant patient.Am. J. Transplant. 20 , 3210-3215 (2020).

17. Thieme, C. J. et al. Robust T Cell Response Toward Spike, Membrane, and Nucleocapsid SARS-CoV-2 Proteins Is Not Associated with Recovery in Critical COVID-19 Patients. Cell Reports Med.1 , 100092 (2020).

18. Anft, M. et al. COVID-19-induced ARDS is associated with decreased frequency of activated memory/effector T cells expressing tissue migration molecule CD11a++. Mol. Ther. 0 , (2020).

19. Cao, Y. et al. Potent neutralizing antibodies against SARS-CoV-2 identified by high-throughput singlecell sequencing of convalescent patients' B cells. Cell (2020) doi:10.1016/j.cell.2020.05.025.

20. Seydoux, E. et al. Analysis of a SARS-CoV-2-Infected Individual Reveals Development of Potent Neutralizing Antibodies with Limited Somatic Mutation. Immunity 53 , 98-105.e5 (2020).

21. Wec, A. Z. et al. Broad neutralization of SARS-related viruses by human monoclonal antibodies. Science (80-. ).369, 731-736 (2020). 
22. Brouwer, P. J. M. et al. Potent neutralizing antibodies from COVID-19 patients define multiple targets of vulnerability.Science (80-. ). 369 , 643-650 (2020).

23. Juno, J. A. et al. Humoral and circulating follicular helper $\mathrm{T}$ cell responses in recovered patients with COVID-19. Nat. Med.26 , 1428-1434 (2020).

24. Ju, B. et al. Human neutralizing antibodies elicited by SARS-CoV-2 infection. Nature 584, 115-119 (2020).

25. Bernasconi, N. L., Traggiai, E. \& Lanzavecchia, A. Maintenance of serological memory by polyclonal activation of human memory B cells.Science (80-. ). 298 , 2199-2202 (2002).

26. Seifert, M. \& Kuppers, R. Human memory B cells. Leukemiavol. 30 2283-2292 (2016).

27. Maruyama, M., Lam, K. P. \& Rajewsky, K. Memory B-cell persistence is independent of persisting immunizing antigen. Nature 407, 636-642 (2000).

28. Traggiai, E. et al. An efficient method to make human monoclonal antibodies from memory B cells: Potent neutralization of SARS coronavirus. Nat. Med. 10 , 871-875 (2004).

29. Dan, J. M. et al. Immunological memory to SARS-CoV-2 assessed for up to 8 months after infection. Science (80-. ). eabf4063 (2021) doi:10.1126/science.abf4063.

30. Hartley, G. E. et al. Rapid generation of durable B cell memory to SARS-CoV-2 spike and nucleocapsid proteins in COVID-19 and convalescence. Sci. Immunol. 5, eabf8891 (2020).

31. Townsend, S. E., Goodnow, C. C. \& Cornall, R. J. Single epitope multiple staining to detect ultralow frequency B cells. J. Immunol. Methods 249 , 137-146 (2001).

32. Boonyaratanakornkit, J. \& Taylor, J. J. Techniques to Study Antigen-Specific B Cell Responses. Frontiers in immunology vol. 101694 (2019).

33. Cossarizza, A. et al. Guidelines for the use of flow cytometry and cell sorting in immunological studies. Eur. J. Immunol. 47 , 1584-1797 (2017).

34. Dunmire, S. K., Verghese, P. S. \& Balfour, H. H. Primary Epstein-Barr virus infection. J. Clin. Virol. $102,84-92(2018)$.

35. Gattinger, P. et al. Antibodies in serum of convalescent patients following mild COVID-19 do not always prevent virus receptor binding. Allergy (2020) doi:10.1111/all.14523.

36. Rodda, L. B. et al. Functional SARS-CoV-2-specific immune memory persists after mild COVID-19. Cell 184, 169-183.e17 (2020).

37. Zhang, Y. et al. Protective humoral immunity in SARS-CoV-2 infected pediatric patients. Cellular and Molecular Immunologyvol. 17 768-770 (2020).

38. Deng, W. et al. Primary exposure to SARS-CoV-2 protects against reinfection in rhesus macaques. Science (80-. ).369 , 818-823 (2020).

Tables

Table 1: List of essential protocol equipment

\begin{tabular}{llll}
\hline Materials & Manufacturer & Country & Cat. Nr. \\
\hline Vacutainer EDTA 10ml (K2E K2EDTA) & Greiner Bio-One & Austria & 455046 \\
BioColl separating solution & Bio\&Sell & Germany & L 6115 \\
Dulbecco's Phosphate Buffered Saline & Gibco & USA & $14190-094$ \\
Transfer pipette 3.5ml & Sarstedt & Germany & 861171001 \\
$50 \mathrm{ml}$ sterile conical tubes & Greiner Bio-One & Austria & 227261
\end{tabular}




\begin{tabular}{llll}
\hline Materials & Manufacturer & Country & Cat. Nr. \\
\hline Trypan Blue & Biochrome & Germany & L6323 \\
Roswell Parm Memorial Institute 1640 + GlutaMax & Gibco & USA & $61870-036$ \\
Penicillin Streptomycin & Gibco & USA & $15140-122$ \\
Fetal bovine serum & Biochrome & Germany & S0115 \\
5ml Polystyrene Round-Bottom Tube & Falcon & USA & 352052 \\
Brilliant stain buffer & BD Horizon & USA & 566349 \\
SARS-CoV-2 (2019-nCoV) Spike S1+S2 ECD-His recombinant Protein & Sinobiological & China & $40589-$ V08B1 \\
Lightning-Link Rapid Fluorescein Labeling Kit & Novus Biologicals & USA & $310-0010$ \\
Lightning-Link Rapid Cy5 Labeling Kit & Novus Biologicals & USA & $342-0010$ \\
SafeSeal tube 0.5ml & Sarstedt & Germany & 72.704 \\
Devices & Manufacturer & Country \\
Standard laboratory equipment & & & \\
CytoFLEX LX & Beckman Coulter & USA & \\
Software & Manufacturer & Country \\
FlowJo version 10.6.2 & BD Biosciences & USA \\
Prism version 7 & GraphPad software & USA \\
R, version 3.6.2 & R-Core-Team & Austria \\
\hline
\end{tabular}

Table 2: B-cell staining panel

B cell panel

\begin{tabular}{lllll}
\hline Antigen & Fluorophore & Clone & Manufacturer & Cat. Nr. \\
CD27 & PE & O323 & BioLegend & 302808 \\
CD20 & BV510 & 2H7 & BioLegend & 302340 \\
IgD & VioBlue & IgD26 & Miltenyi Biotec & $130-123-319$ \\
CD19 & BV605 & HIB19 & BioLegend & 302244 \\
CD38 & BV650 & HB-7 & BioLegend & 356620 \\
CD3 & BV785 & OKT3 & BioLegend & 317330 \\
CD14 & APC-Cyanine 7 & M5E2 & BioLegend & 301820 \\
DAPI & & & ThermoScientific & 62248 \\
\hline
\end{tabular}

Table 3: Baseline characteristics and protocol parameters applied for COVID-19 patient and healthy donor validation cohort.

COVID-19 patient cohort

\begin{tabular}{lll}
\hline ID & Severity [mild, severe] & Age [years] \\
P01 & mild & 30 \\
P02 & mild & 27 \\
P03 & mild & 53 \\
P04 & mild & 51 \\
P05 & mild & 74 \\
P06 & mild & 55 \\
P07 & mild & 77 \\
P08 & mild & 81 \\
P09 & mild & 61 \\
P10 & mild & 41 \\
P11 & mild & 63
\end{tabular}




\begin{tabular}{|c|c|c|}
\hline P12 & mild & 57 \\
\hline P13 & mild & 35 \\
\hline P14 & mild & 47 \\
\hline P15 & moderate & n.a. \\
\hline P16 & mild & 27 \\
\hline P17 & mild & 66 \\
\hline P18 & mild & 59 \\
\hline P19 & mild & 71 \\
\hline P20 & moderate & 79 \\
\hline P21 & severe & 43 \\
\hline P22 & severe & 66 \\
\hline P23 & moderate & 51 \\
\hline P24 & moderate & 60 \\
\hline P25 & severe & 88 \\
\hline P26 & moderate & 82 \\
\hline Median & Median & 59 \\
\hline Min & Min & 27 \\
\hline $\operatorname{Max}$ & $\operatorname{Max}$ & 88 \\
\hline Healthy donor cohort & Healthy donor cohort & Healthy donor cohort \\
\hline H01 & NA & 45 \\
\hline H02 & NA & 58 \\
\hline H03 & NA & 48 \\
\hline H04 & NA & 28 \\
\hline H05 & NA & 27 \\
\hline H06 & NA & 49 \\
\hline H07 & NA & 26 \\
\hline H08 & NA & 48 \\
\hline H09 & NA & 60 \\
\hline H10 & NA & 60 \\
\hline H11 & NA & 40 \\
\hline H12 & NA & 49 \\
\hline H13 & NA & 60 \\
\hline H14 & NA & 46 \\
\hline Median & Median & 48 \\
\hline Min & Min & 26 \\
\hline $\operatorname{Max}$ & $\operatorname{Max}$ & 60 \\
\hline $\begin{array}{l}\text { COVID-19 vs. Healthy donor cohort } \\
\mathrm{P}=\end{array}$ & $\begin{array}{l}\text { COVID-19 vs. Healthy donor cohort } \\
\mathrm{P}=\end{array}$ & $\begin{array}{l}\text { COVID-19 vs. Healthy } \\
0.0323\end{array}$ \\
\hline
\end{tabular}

Figure legends

Figure 1: Conceptual overview of the protocol for SARS-CoV-2 spike specific Memory B-cell detection . *Detailed B-cell staining panel is provided in Table 2. Abbreviations: EDTA = Ethylenediaminetetraacetic acid, $\mathrm{PBMC}=$ Peripheral blood mononuclear cell.

Figure 2: Detection of SARS-CoV-2 specific B cell subsets. (a) Gating strategy for the detection of B cell subsets. (b) Representative example for the detection of dual-labelled SARS-CoV-2 S-protein binding B-cells and quantification of antigen-specific B cell subsets. Comparison of samples without fluorochromecoupled SARS-CoV-2 protein ("No S-protein") and SARS-CoV-2 S-protein in concentrations of 1:500 and 1:1000 with and without excess unlabelled protein to block B cell receptors.

Figure 3: Detection of COVID-19 specific BMEMORY cells using spike (S)-protein. Periph- 
eral blood mononuclear cells of convalescent COVID-19 patients $(\mathrm{n}=26)$ and healthy donors $(\mathrm{n}=14)$ were incubated with fluorochrome labeled SARS-CoV-2 S-protein with or without excess unlabelled protein to

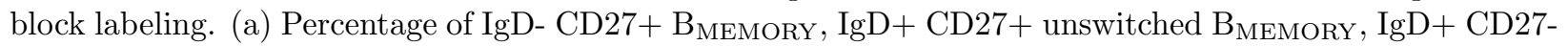
$\mathrm{B}_{\mathrm{NAIVE}}$ and CD27++ CD38++ plasmablasts within the entire B-cell population analysed for COVID-19 disease and healthy cohorts. Bars show median with interquartile range. (b) Correlation of fluorochrome labelled SARS-CoV-2 S-protein binding B cells and anti-S1/S2-IgG. $n=21$ samples of 19 COVID-19 patients (two patients with two samples collected at different time points) and 14 healthy donors. Analysis was performed with Spearman's rank coefficient, $r h o=0.42, \mathrm{P}=0.011$. (c)-(f) Frequencies of S-protein binding $B_{\text {Memory }}(\mathrm{c})$, B Baive (d), plasmablasts (e) and unswitched B Bemory cells (f) after staining with preincubation of unlabelled Covid-19 antigen (blocked, left) and without preincubation (not blocked staining, right) samples. $n=26$ COVID-19 patients and $n=14$ healthy donors. Statistical comparison was done with two-way repeated measurements ANOVA and Sidak's multiple comparisons test. (g) Correlation of fluorochrome labelled SARS-CoV-2 S-protein binding B cells and days after COVID-19 diagnosis. $\mathrm{n}=25$ samples of 23 COVID-19 patients (two patients with two samples collected at different time points). The analysis was performed with Spearman's rank coefficient, $\mathrm{rho}=-0.34, \mathrm{P}=0.095$. 


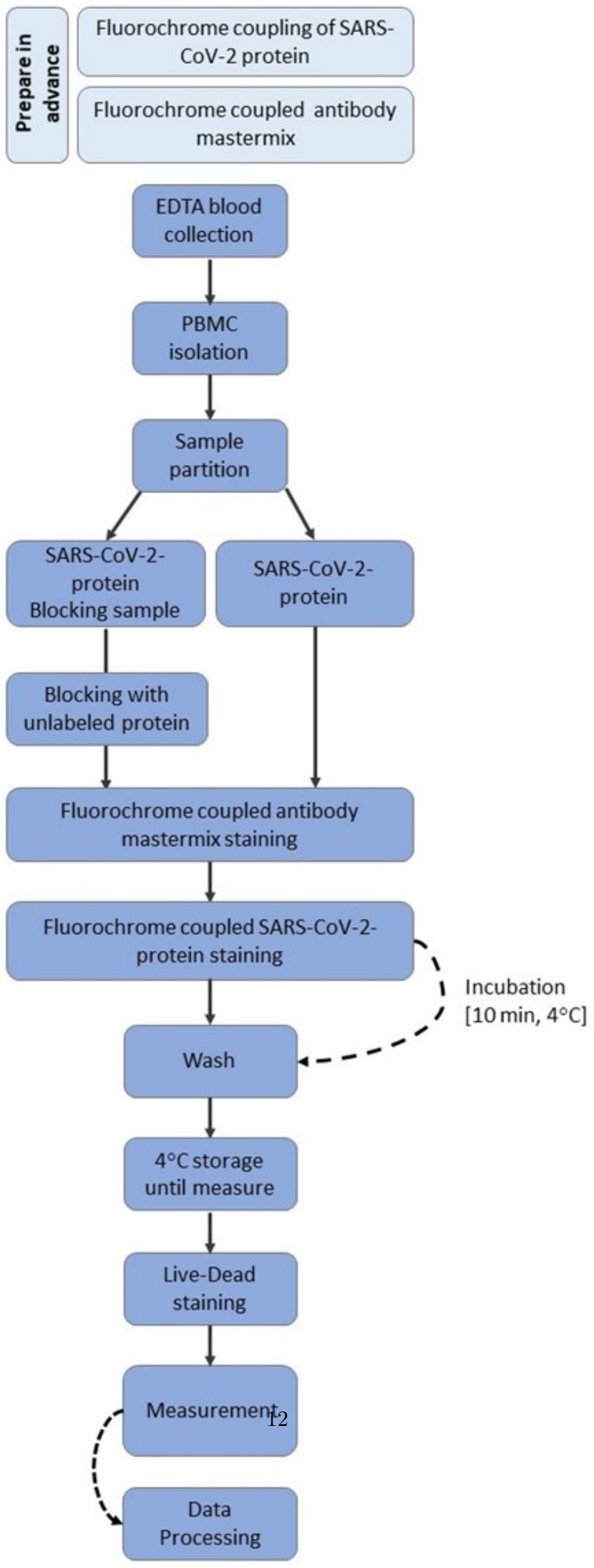



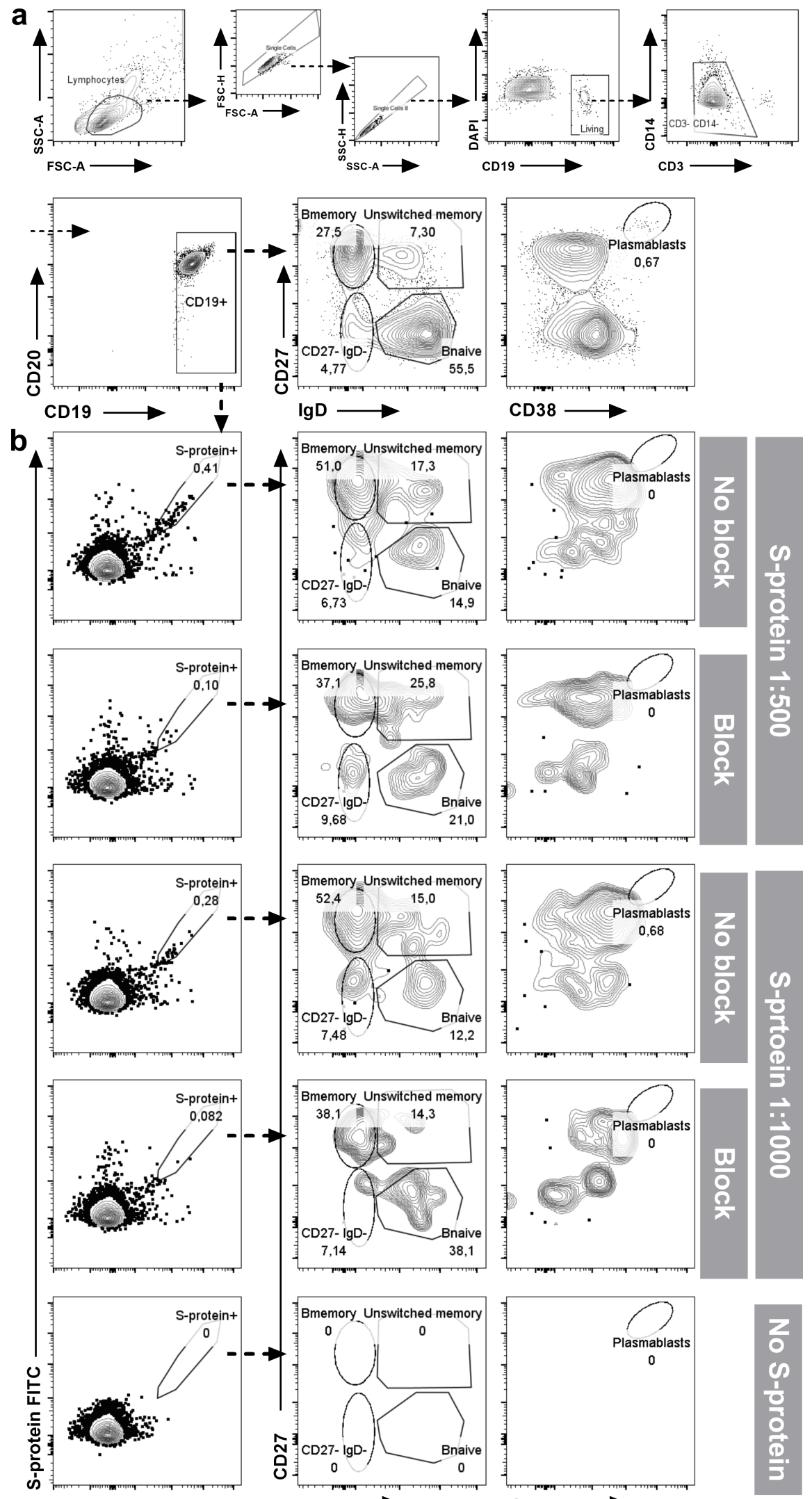

S-protein Cy5
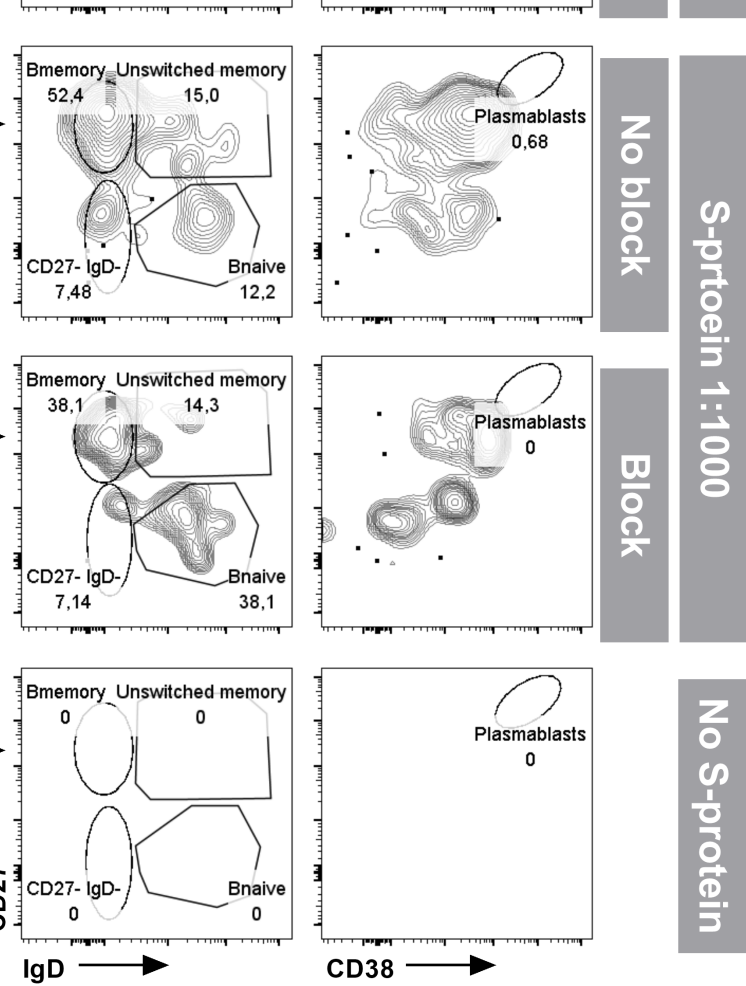
a
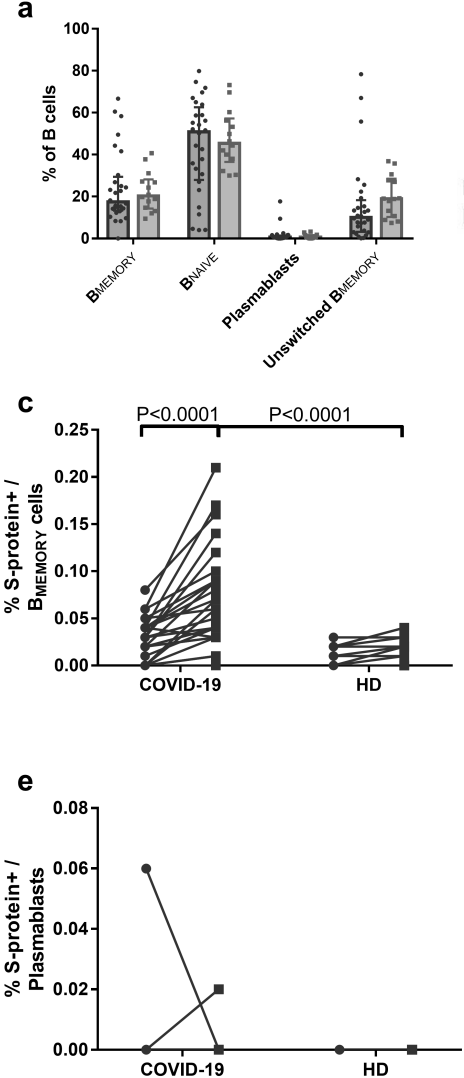

b
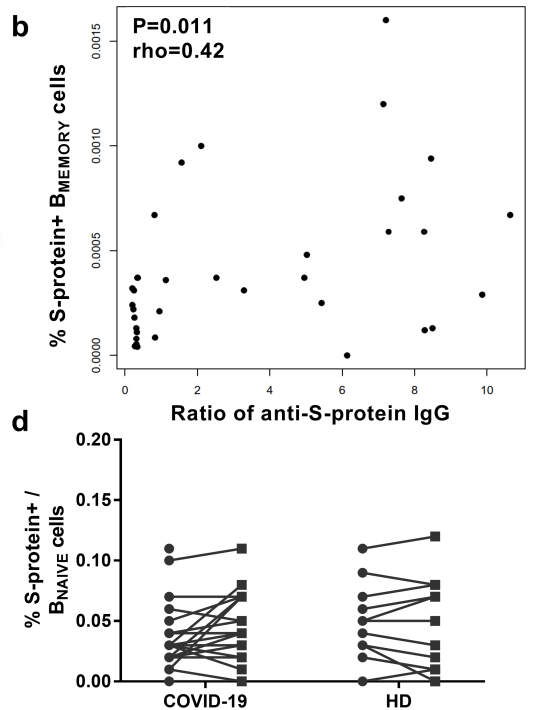

- Block

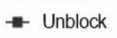

f

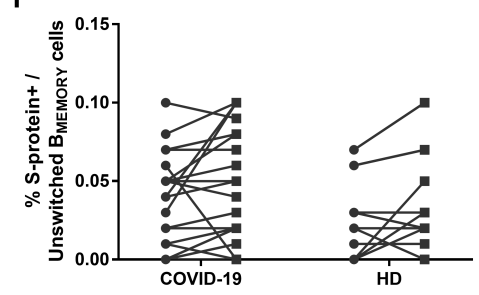

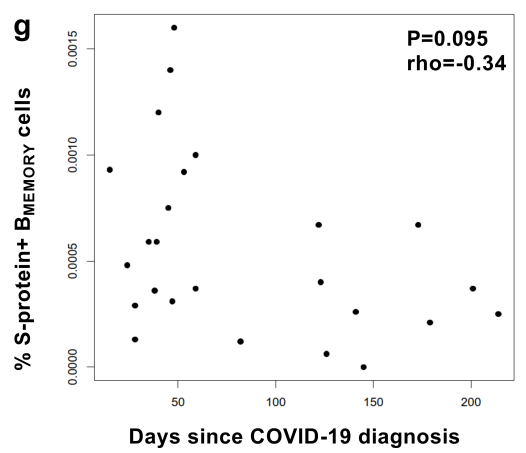

